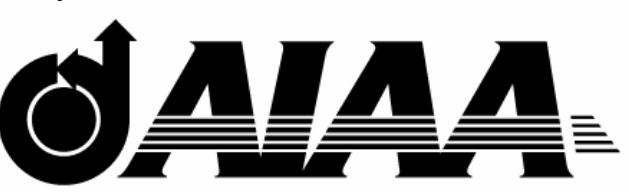

\title{
AIAA 2003-1103
}

DES GRID RESOLUTION ISSUES FOR

VORTICAL FLOWS ON A DELTA WING AND

AN F-18C

Scott A. Morton, Matthew B. Steenman, Russell M. Cummings, and James R. Forsythe The Department of Aeronautics United States Air Force Academy USAF Academy, CO 80840

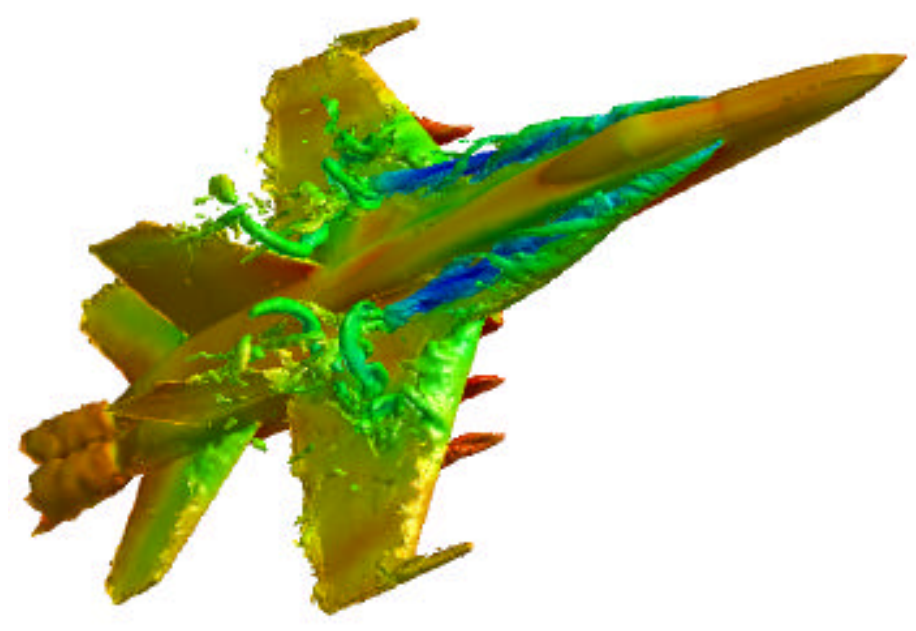

\section{$41^{\text {st }}$ Aerospace Sciences Meeting \& Exhibit 6-9 January, 2003 Reno, Nevada}

For permission to copy or to republish, contact the copyright owner named on the first page.

For AIAA-held copyright, write to AIAA Permissions Department,

1801 Alexander Bell Drive, Suite 500, Reston, VA, 20191-4344. 
AIAA 2003-1103 


\title{
DES GRID RESOLUTION ISSUES FOR VORTICAL FLOWS ON A DELTA WING AND AN F-18C
}

\author{
Scott A. Morton*, Matthew B. Steenman ${ }^{\#}$, Russell M. Cummings \% , and James R. Fors ythe * \\ United States Air Force Academy, Department of Aeronautics \\ 2410 Faculty Drive, Suite 108 \\ USAF Academy, CO 80840-6400, USA.
}

\begin{abstract}
An assessment of unstructured grids for use in Detached-Eddy Simulations (DES) of vortical flowfields over two configurations, a 70 degree delta wing and an F-18C are presented. The role of the grid in detached eddy simulations of vortical flowfields, including complex features such as vortex breakdown, is assessed on a delta wing with comparison to wind tunnel data. Adaptive mesh refinement is applied to the delta wing grid to improve the focus region aft of the vortex breakdown where massively separated flow exists and unsteady pressures are generated that could impact the loads on vertical tails of more complex configurations. The adaptively refined mesh is compared to the baseline mesh to determine the advantage of the adaptive mesh refinement approach for vortex breakdown. The focus region grid resolution is then applied to an $\mathrm{F}-18 \mathrm{C}$ in the region of the vortex generat ed from the leading edge extension (LEX). The resulting unsteady tail loads are compared to flight test data from the NASA F-18 HARV database. This paper represents one of the first times adaptive mesh refinement will be applied to a detached eddy simulation of a flight vehicle configuration.
\end{abstract}

\section{INTRODUCTION}

Many of todays military vehicles exhibit vortex dominated flowfields. At a recent NATO Air Vehicle Technology conference, D. A. Lovell presented a review of "Military Vortices," where he discussed the declining research budget in this area and the importance of understanding the phenomena. He classified vortex flows into three categories, "those designed into a vehicle to improve performance, those which cannot be avoided and whose adverse affects must be minimized, and those that were not expected to occur." "He gives examples of many of these vortex dominated flowfields: tip vortices on wings having low sweep, leading edge extension vortices from the F-18 and F-16 aircraft, foreplanes on the Rafale, and flow over the MK-82 bomb, to name just a few. He also discusses the fact that governments are relying ever increasingly on the aerospace industry to perform research. Since the aerospace industry concentrates on cruise conditions for optimization of commercial aircraft, these vortical flowfields common in military aircraft are losing their place in research budgets. This is occurring at a time when the three largest US fighter development programs (F/A$18 \mathrm{E} / \mathrm{F}, \mathrm{F}-22$, and F-35) incorporate twin tail configurations and high angle-of-attack maneuvering.

The F-18 High Angle of Attack Research Vehicle (HARV; see Fig. 1) has proven to be an excellent source of data for researchers working on high angle of attack flowfields. ${ }^{3,4,42}$ Extensive flight testing of the HARV has been conducted that provides a rich source of flow visualization, surface pressures, and aeroelastic information. The F-18 utilizes wing leading edge extensions (LEX) to generate

\footnotetext{
* Associate Professor of Aeronautics, AIAA Associate Fellow.

\# USAF Academy Undergraduate Student, AIAA Student Member.

${ }^{\%}$ Distinguished Visiting Professor, AIAA Associate Fellow.

\& Associate Professor of Aeronautics, AIAA Senior Member.

This paper is declared a work of the US government and is not subject to copyright protection in the United States.
}

vortices which enhance the wing lift, and the twin vertical tails are canted to intercept the strong vortex field and increase maneuverability. At large incidence, the LEX vortices breakdown upstream of the vertical tails, resulting in a loss of yaw control power and severe aeroelastic effects. ${ }^{5}$ This tail buffet phenomenon was reduced by using extensive flight tests to design a LEX fence. The ultimate goal of computationally modeling the flowfield shown in Fig. 1 would be to accurately simulate the aeroelastic impact of the LEX vortices on the twin vertical tails. The current level of simulation technology, however, has not allowed for accurate prediction of vortex breakdown, and the unsteady flow downstream of breakdown, at fight Reynolds numbers. Because of this, researchers have used simpler geometries, such as slender forebodies and delta wings, to improve their simulation capabilities.

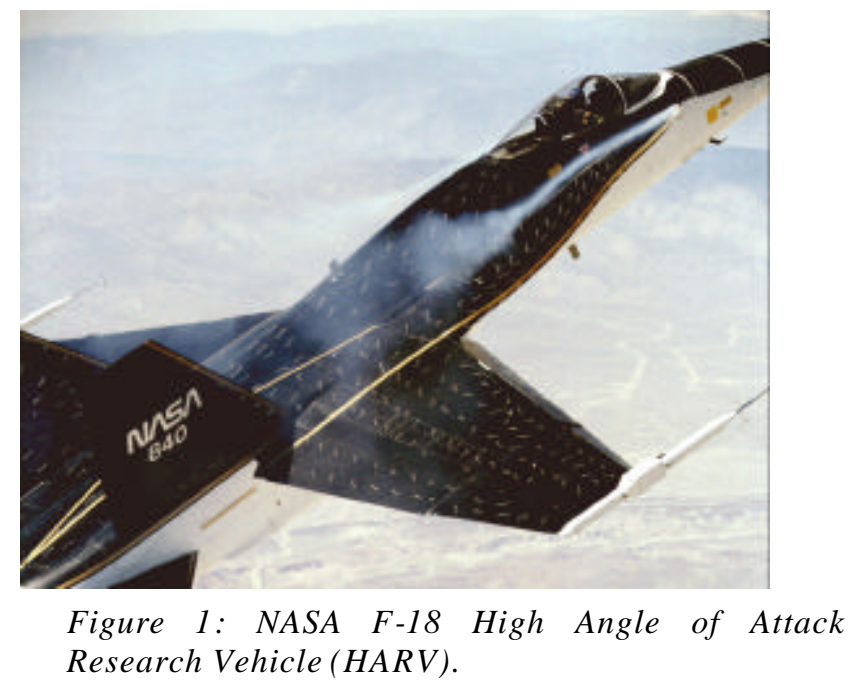

The delta wing vortex breakdown phenomena has been studied extensively since Henri Werlé first photographed it in 1954, during water tunnel tests of a slender delta wing model at Onera. ${ }^{6}$ This work was quickly confirmed by Peckham and Atkinson, ${ }^{7}$ Elle $^{8}$ and Lambourne and Bryer ${ }^{9}$ 
and spawned a large number of experimental, computational and theoretical studies which continue today. These investigations led to the development of several theories governing vortex breakdown, although none have been universally accepted ${ }^{10-14}$. Despite this lack of a unified theoretical interpretation, several forms of vortex breakdown have been identified ${ }^{12,15}$ (i.e. bubble, helical, etc.), and the global characteristics of the phenomena are understood. During the breakdown process, the mean axial velocity component rapidly decreases until it reaches a stagnation point and/or becomes negative on the vortex axis. This stagnation point, called the breakdown location, is unsteady and typically oscillates about some mean position along the axis of the vortex core. ${ }^{16,17}$ As angle of attack is increased, the mean vortex breakdown location moves upstream over the delta wing (from the trailing edge towards the apex).

The primary vortex over a slender delta wing at angle of attack is principally inviscid. Unfortunately, the location of the vortex is strongly affected by a secondary vortex formed by the inter-relationship between the surface boundary layer and the primary vortex. In addition, the vortex breakdown phenomenon creates turbulent kinetic energy that must be modeled properly or resolved. Many turbulence models create orders of magnitude too much turbulent eddy viscosity in the primary vortex core which significantly alters the flowfield and in some cases eliminates breakdown observed experimentally at high Reynolds numbers. For these reasons, an accurate prediction of the flowfield over a slender delta wing at high angles of attack and high Reynolds numbers (as well as military aircraft exhibiting vortex breakdown) must model the boundary layer, primary and secondary vortex, and turbulent kinetic energy correctly.

While advances have taken place in areas such as grid generation and fast algorithms for solutions of systems of equations, CFD has remained limited as a reliable tool for prediction of inherently unsteady flows at flight Reynolds numbers. Current engineering approaches for prediction of unsteady flows are based on solution of the Reynoldsaveraged Navier-Stokes (RANS) equations. The turbulence models employed in RANS methods necessarily model the entire spectrum of turbulent motions. While often adequate in steady flows with no regions of reversed flow, or possibly exhibiting shallow separation, it appears inevitable that RANS turbulence models are unable to accurately predict flows characterized by massive separation. Unsteady, massively separated flows are characterized by geometry-dependent and three dimensional turbulent eddies. These eddies, arguably, are what defe at RANS turbulence models, of any complexity.

To overcome the deficiencies of RANS models for predicting massively separated flows, Spalart et.al. ${ }^{28}$ proposed Detached-Eddy Simulation (DES) with the objective of developing a numerically feasible and accurate approach combining the most favorable elements of RANS models and Large Eddy Simulation (LES). The primary advantage of DES is that it can be applied at high Reynolds numbers as can Reynolds-averaged techniques, but also resolves geometry-dependent, unsteady threedimensional turbulent motions as in LES. The unstructured finite-volume solver Cobalt ${ }^{20}$ has been used in conjunction with DES successfully on a number of complex problems, including a supersonic base flow ${ }^{21}$, delta wing vortex breakdown $^{22}$, a square with rounded corners ${ }^{23}$, the F-15E at high angle of attack ${ }^{24}$, and the F/A-18E with unsteady shock buffet ${ }^{25}$.

The specific aim of this work is to document the effects of grid resolution on detached eddy simulations of slender delta wings at high Reynolds number and then apply the necessary grid requirements to the F-18C (without the

LEX fence) at a condition consistent with vortex breakdown. Computations are made for the $\mathrm{F} 18 \mathrm{C}$ at $\alpha=30^{\circ}, \quad M_{\infty}=0.2755$, and $\operatorname{Re}_{\bar{c}}=13.9 \times 10^{6}$ which determine the importance of highly refined grids (including autuomatic mesh refinement) on the accurate prediction of complex vortical flowfields. Comparisons are made between steady Reynolds-averaged NavierStokes (RANS), unsteady Reynolds-averaged NavierStokes (U-RANS), and Spalart-Allmaras DES (SADES), and the resulting predictions are compared with available flight test data for the F-18 HARV.

\section{NUMERICAL METHOD}

In this section a brief description of the numerical method is provided. Full details of the computational scheme and the solution method are presented in Reference [20]. The two configurations of interest are Onera's sharp-edged, $70^{\circ}$ sweep angle $(\Lambda)$ delta wing with a root chord (c) of $950 \mathrm{~mm}^{2,17}$ and the $\mathrm{F}-18 \mathrm{C}$ with leading and trailing edge flaps set to 0 , a diverter slot with flow through the LEX, mass flow through the inlet and nozzle consistent with the engine at full throttle, and no LEX fence.

Solutions for both configurations were computed with the commercial version of Cobalt developed by Cobalt Solutions. Cobalt solves the unsteady, three-dimensional, compressible Navier-Stokes equations on a hybrid unstructured grid. The code has several choices of turbulence models, including Spalart Almaras (SA), SA with approximate rotation corrections of Dacles-Mariani (ASARC), and Mentor's Shear Stress Transport (SST) RANS, as well as DES versions of SA and SST. All simulations were computed on unstructured meshes with prisms in the boundary layer and tetrahedra elsewhere on half-span surface geometries. The computational meshes were generated with the software packages GridTool ${ }^{26}$ and VGRIDns $^{27}$. 


\section{Turbulence Models}

For simulation of turbulent flows, the governing equations are suitably averaged, yielding turbulent stresses that require a model. A Boussinesq approximation is invoked in the momentum equations and the turbulent eddy viscosity $\left(\mu_{t}\right)$ is used to relate the stresses to the strain rate. The turbulent heat flux is also modeled using a gradient-transport hypothesis, requiring specification of a turbulent thermal conductivity, $k_{t}$. The Reynolds analogy is applied and the turbulent heat flux is modeled using a constant turbulent Prandtl number of 0.9. Using turbulent eddy viscosity and turbulent conductivity, the variable $\mu$ is replaced by $\left(\mu+\mu_{t}\right)$ and $k$ is replaced by $\left(k+k_{t}\right)$ in the governing equations.

\section{Spalart-Allmaras Turbulence Model}

The Spalart-Allmaras ${ }^{18}$ (SA) one equation model solves a single partial differential equation for a working variable $\tilde{v}$ which is related to the turbulent viscosity. The differential equation is derived by "using empiricism and arguments of dimensional analysis, Galilean invariance and selected dependence on the molecular viscosity."18 The model includes a wall destruction term that reduces the turbulent viscosity in the laminar sublayer. The model takes the form,

$$
\begin{aligned}
\frac{D \tilde{\mathrm{v}}}{D t} & =c_{b 1} \tilde{S} \tilde{\mathrm{v}}-c_{w 1} f_{w}\left[\frac{\tilde{\mathrm{v}}}{d}\right]^{2} \\
& +\frac{1}{\sigma}\left[\nabla \cdot((v+\tilde{\mathrm{v}}) \nabla \tilde{\mathrm{v}})+c_{b 2}(\nabla \tilde{\mathrm{v}})^{2}\right]
\end{aligned}
$$

The turbulent kinematic viscosity is obtained from,

$$
v_{t}=\frac{\mu_{t}}{\rho}=\tilde{v} f_{v 1}, \quad f_{v 1}=\frac{\chi^{3}}{\chi^{3}+c_{v 1}^{3}}, \quad \chi \equiv \frac{\tilde{v}}{v}
$$

where $S$ is the magnitude of the vorticity given by

$$
S=|\omega|=|\nabla \times(u \hat{i}+v \hat{j}+w \hat{k})|,
$$

and the modified vorticity is,

$$
\begin{gathered}
\tilde{S} \equiv S+\frac{\tilde{v}}{\kappa^{2} d^{2} f_{v 2},} \\
f_{v 2}=1-\frac{\chi}{1+\chi f_{v 1}},
\end{gathered}
$$

where $d$ is the distance to the closest wall. The wall destruction function $f_{w}$ is,

$$
f_{w}=g\left[\frac{1+c_{w 3}^{6}}{g^{6}+c_{w 3}^{6}}\right]^{\frac{1}{6}},
$$

and

$$
g=r+c_{w 2}\left(r^{6}-r\right), \quad r \equiv \frac{\tilde{v}}{\tilde{S} \kappa^{2} d^{2}} .
$$

The turbulent viscosity is obtained from the turbulent kinematic viscosity by $\mu_{t}=\rho v_{t}$.

The model coefficients are,

$$
\begin{array}{ccc}
c_{b 1}=0.1355 & \sigma=2 / 3 & c_{b 2}=0.622 \\
\kappa=0.41 & c_{w 1}=c_{b 1} / \kappa^{2}+\left(1+c_{b 2}\right) / \sigma & c_{w 2}=0.3 \\
c_{w 3}=2 & c_{v 1}=7.1 &
\end{array}
$$

\section{Detached-Eddy Simulation}

Detached-Eddy Simulation (DES) was proposed by Spalart et $\mathrm{al}^{28}$ The motivation for this approach was to combine large-eddy simulation (LES) with the best features of Reynolds-averaged Navier-Stokes (RANS) methods. RANS methods have demonstrated an ability to predict attached flows very well with a relatively low computational cost. LES methods have demonstrated an ability to compute seperated flowfields accurately, but at a tremendous cost for configurations with boundary layers. Spalart's DES method is a hybrid of LES and RANS, which combines the strengths of both methods.

The DES model was originally based on the SpalartAllmaras one equation RANS turbulence model (detailed above) with a more detailed presentation in Ref. [18]. The wall destruction term presented above is proportional to $(\tilde{v} / d)^{2}$, where $d$ is the distance to the wall. When this term is balanced with the production term, the eddy viscosity becomes proportional to $\hat{S} d^{2}$ where $\hat{S}$ is the local strain rate. The Smagorinski LES model varies its sub-grid scale (SGS) turbulent viscosity with the local strain rate, and the grid spacing: $v_{S G S} \propto \hat{S} \Delta^{2}$, where $\Delta=\max (\Delta x, \Delta y, \Delta z)$. If $\boldsymbol{d}$ is replaced with $\Delta$ in the wall destruction term, the SA model will act as a Smagorinski LES model.

To exhibit both RANS and LES behavior, $\boldsymbol{d}$ in the SA model is replaced by

$$
\tilde{d}=\min \left(d, C_{D E S} \Delta\right) \text {. }
$$

When $d<<$, the model acts in a RANS mode and when $d \gg \Delta$ the model acts in a Smagorinski LES mode. Therefore the model switches into LES mode when the grid is locally refined.

DES was implemented in an unstructured grid method by Forsythe et. al. ${ }^{29}$ They determined the $C_{D E S}$ constant should be 0.65 , consistent with the structured grid implementation of Spalart et. al. ${ }^{28}$ when the grid spacing $\Delta$ was taken to be the longest distance between the cell center and all of the neighboring cell centers.

A Newton sub-iteration method is used in the solution of the system of equations to improve time accuracy of the point implicit method and approximate Jacobians. In the calculations presented below, a typical number of three Newton sub-iterations is used for all time-accurate cases. 


\section{Grid Generation}

Spalart $^{19}$ described the process of grid design and assessment for DES, defining important regions of the solution and offering guidelines for grid densities within each region. The "Young-Person's Guide" (YPG) forms a basis for interpretation of many of the results presented below. One of the traditional motivations for using unstructured grids has been the ability to rapidly create grids around complex geometries. There are other positive attributes of unstructured grids that are relevant to DES. Most notably, it is possible to concentrate points in the region of interest (i.e. the vortex core or aft of breakdown) and rapidly coarsen the grid away from these areas. This region of interest was termed the "focus region" in the YPG. Another advantage exploited in the present study is the isotropic cells generated in the LES region by most unstructured grid generation packages. The YPG reference describes the desirability of having isotropic grid cells in the focus region in which unsteady, time-dependent, features are resolved. For this reason, unstructured grids are good candidates for use in DES because near isotropy of the grid cells in the LES region is assured by most grid generation packages.

Morton et al. ${ }^{30}$ applied the YPG guidelines to three massively separated flows of interest: forebody in a crossflow, flow over a delta wing at $27^{\circ}$ angle of attack (also the subject of this work), and the flow over an F-15E at $65^{\circ}$ angle of attack. In the latter two cases an extensive grid sensitivity study was performed by systematically varying the grid by a scale parameter allowing a very consistent analysis of grid effects when using the DES method of computing massively sesparated flows. A further refinement of the delta wing grids was presented in Ref. [31] as well as the first use of adaptive mesh refinement with DES.

Another important grid technology that is particularly well suited for DES is adaptive mesh refinement. Pirzadeh ${ }^{32}$ presented a method based on a tetrahedral unstructured grid technology developed at NASA Langley Research Center with application to two configurations with vortex dominated flowfields. The large improvement of the adapted solutions in capturing vortex flow structures over the conventional unadapted results was demonstrated by comparisons with wind tunnel data. Pirzadeh showed the numerical prediction of these vortical flows was highly sensitive to the local grid resolution and he also stated that grid adaptation is essential to the application of CFD to these complicated flowfields. His most successful computations were performed using an inviscid method due to the inadequacies of standard turbulence models in computing these complicated flowfields. Pirzadeh's method is applied to the Onera delta wing configuration and the F-18C in the current study. A mean flow solution on a baseline grid is used to create an adaptively refined mesh and the new grid used with DES to compute the unsteady flowfield for these two configurations. All meshes of the current study produced average $\mathrm{y}^{+}$values less than 1 .

\section{RESULTS}

This section presents results of the numerical simulations for the delta wing with comparison of these simulations to the Onera experimental data set, as well as the F-18C with comparison to the NASA HARV flight-test data. The results section will be separated into subsections for the two configurations of interest.

\section{Onera $70^{\circ}$ Sweep Delta Wing}

All delta wing cases were run at a freestream velocity of $24 \mathrm{~m} / \mathrm{s}$, an angle of attack of $27^{\circ}$, a Mach number of 0.069 , and other freestream conditions consistent with a Reynolds number of 1.56 million. No attempt was made to model transition from laminar to turbulent flow on the delta wing. In all cases, the spatial and temporal operators were second-order accurate. Typical unsteady simulations were run for 10,000 time steps with an iteration plus three subiterations per time step. Frequency domain analysis was performed on the last 9,000 of 10,000 iterations to eliminate the effects of transients. Averaged quantities are provided based on the the latter 8,000 iterations.

When computing solutions for unsteady flowfields such as vortex breakdown, it is important to determine the degree to which the solution is time accurate and grid insensitive. Morton et. al. ${ }^{2}$ presented an extensive time step study using the current method and the baseline grid. They determined the appropriate time step, nondimensionalized by the freestream velocity and the root chord, for the baseline grid of the current study was 0.0025 with three Newton subiterations. A comprehensive grid sensitivity study was also performed and documented in Ref. B0], where the baseline grid was one in a series of four grids with 1.2, 2.7, 6.7 and 10.7 million cells. This series of grids was produced using a consistent scale factor of $1 / \sqrt{2}$ to make each successive grid. The current study compares the baseline grid with 2.7 million cells with a 3.2 million cell grid produced with adaptive mesh refinement (AMR) of the baseline grid using the Refinemesh software of Pirzadeh ${ }^{32}$.

An AMR grid is produced by using a flowfield solution on the baseline grid to produce an iso-surface of vorticity. All of the cells within the iso-surface of vorticity are removed and the VGRIDns software is used to re-grow a grid in this region with a user defined scale factor increase in the number of points in the re-growth region. In the current study, twice as many points were added to the focus region. This was done for two different levels of vorticity successively, resulting in a grid with 3.2 million cells.

Figures 2 and 3 depict planes perpendicular to the delta wing surface at four chord-wise locations, $500 \mathrm{~mm}$, $600 \mathrm{~mm}, 700 \mathrm{~mm}$, and $800 \mathrm{~mm}$. The viscous layers of prisms are evident in both figures. Figure 3 clearly demonstrates the ability of the AMR technique to 
concentrate points in the vortex core and leading-edge shear-layer "focus" regions.

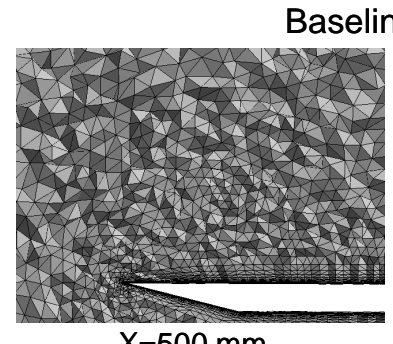

Baseline Grid

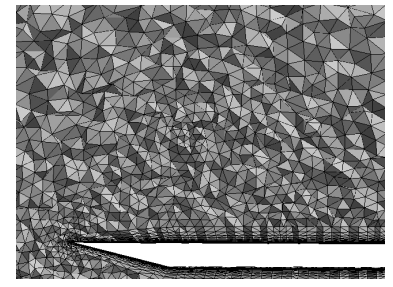

$\mathrm{X}=700 \mathrm{~mm}$

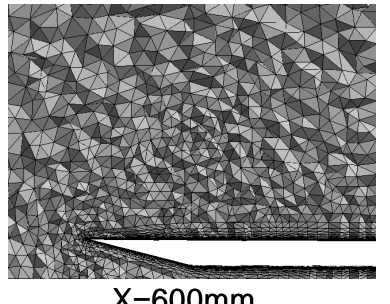

$\mathrm{X}=600 \mathrm{~mm}$

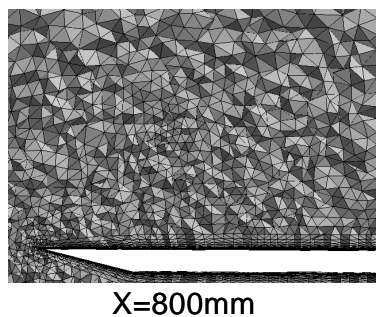

Figure 2: Baseline grid with 2.7 million cells, 19 viscous layers with the first 13 of the tetrahedron layers combined into prisms.

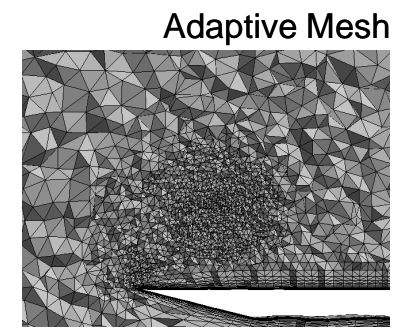

$\mathrm{X}=500 \mathrm{~mm}$

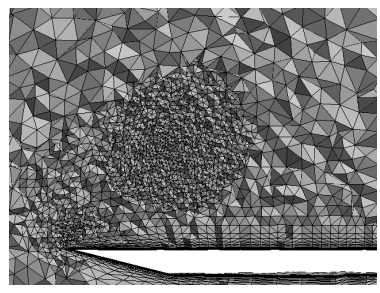

$\mathrm{X}=700 \mathrm{~mm}$

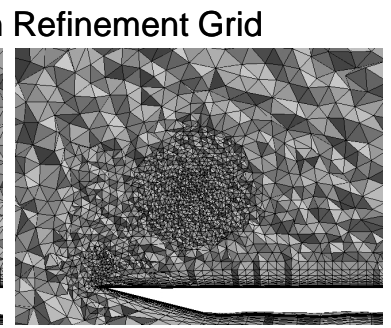

$\mathrm{X}=600 \mathrm{~mm}$

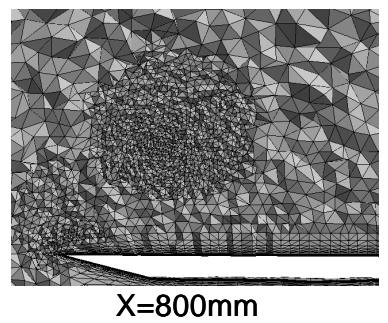

Figure 3: AMR grid with 3.2 million cells, 19 viscous layers with the first 13 of the tetrahedron layers combined into prisms (adapted from baseline grid).

Figure 4 depicts the instantaneous flow field solutions from the baseline and AMR semi-span grids, mirrored across the symmetry plane, after 10,000 iterations. Isosurfaces of vorticity magnitude equal to $750 \mathrm{~s}^{-1}$ are presented, colored by the spanwise component of vorticity. In the baseline grid solution (Fig. 4a) there are several notable features. The iso-surface of vorticity highlights the existence of a coherent leading edge vortex that rapidly changes shape at the breakdown location. Aft of breakdown, helical structures are formed that wind in an opposite direction of the core vortex. The secondary vortex is evident in the top view close to the leading edge. Also, alternating pairs of coherent vortices along the trailing edge, oriented in the spanwise direction, are evident.
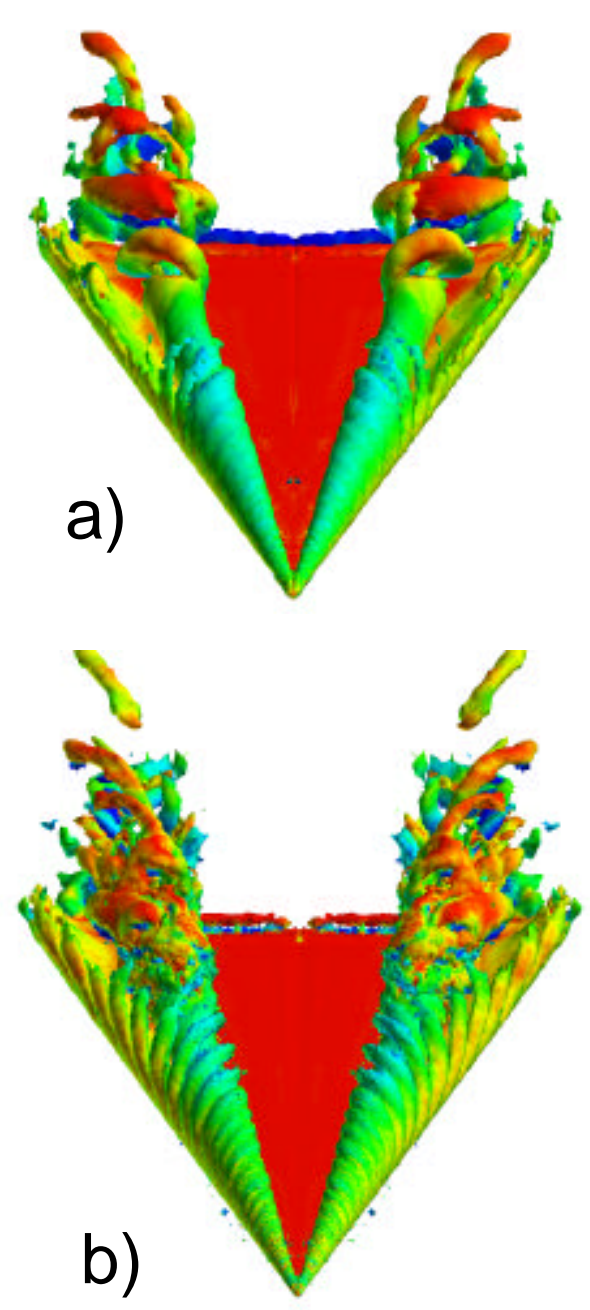

Figure 4: Instantaneous solutions showing an isosurface of vorticity magnitude colored by pressure for a) the baseline grid and b) the AMR grid..

The AMR grid solution depicted in Fig 4b displays all of the features of the baseline grid solution but with some additional features, primarily in the pre-breakdown region. There are vortical substructures observed that are very coherent and stationary in time and even persist downstream of the breakdown position. These vortical substructures have been observed experimentally and documented in Ref.'s 33-41]. The trailing-edge vortices are also evident but are formed by smaller 3-D structures eminating from the trailing edge and rolling up into the strong coherent 2-D spanwise vortices. Consistent with the fact that the core of the vortex computed with AMR is much more refined than the baseline grid, there is a tremendous amount of three-dimensional structures in the region of the core, post-breakdown.

Figure 5 depicts planes of axial vorticity experimentally obtained by Laser Doppler Velocimetry for the Onera delta 
wing configuration at the same conditions as the computations. Details of the experiment, as well as additional data, are presented by Mitchell et.al. ${ }^{41}$. The results shown in Fig. 5 demonstrate the evolution of the discrete substructures of vorticity around the vortex core. The substructures appear to roll around the vortex core as they evolve in the downstream direction. It is interesting to note that the substructures follow a helical trajectory around the vortex core and the spacing (frequency) between the substructures appears relatively constant, confirming the observations of Washburn and Visser ${ }^{32}$. Additionally, the substructures remain coherent even in the post-breakdown region of the flowfield as is observed in the computations (Fig. 4b).

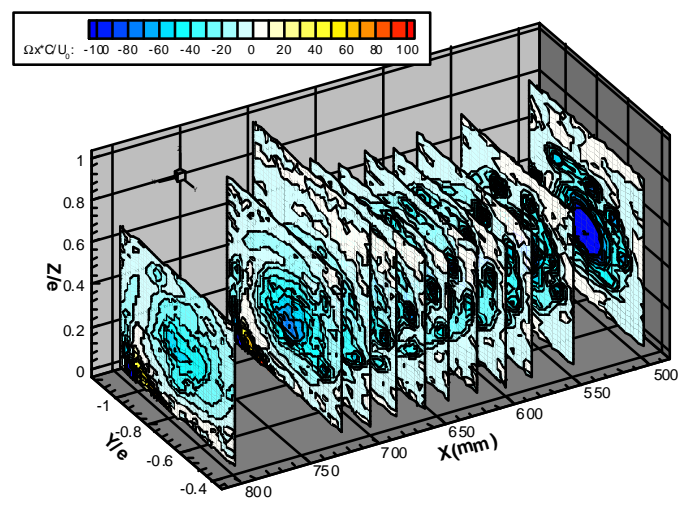

Figure 5: $L D V$ results of axial vorticity measured in 14 different planes perpendicular to the leeward surface of the $70^{\circ}$ delta wing at $\alpha=27^{\circ}$ and $R_{c}=$ $1.56 \times 10^{6}$ demonstrating the existence and form of the vortical substructures. Freestream velocity from right to left.( from Ref. [31]).

In the experimental results shown in Fig. 5, the trace of a helix is obtained (defined as $\mathrm{L} / 2 \pi \mathrm{r}$; where $\mathrm{L}$ is the longitudinal distance for one rotation and $r$ is the radius of the helix). Using the data represented in Figs. $4 b$ and 5, the helix trace is computed and presented in Table 1. As seen in Table 1, the comparison between the AMR grid solution and the experiment is quite good. In addition, the vortex breakdown postion was compared to experiment and found to lie within the scatter of the experimental data ${ }^{2}$. The close correlation of the traces of the substructures between the experimental results and the DES solutions shown in Table 1 and the vortex breakdown position are additional indications of the accurate predictive capabilities of this DES method for vortical and highly separated flows.

\begin{tabular}{|l|l|}
\hline & Helix Trace $(\mathrm{L} / 2 \pi \mathrm{r})$ \\
\hline ONERA experimental results & $1.06-1.32$ \\
\hline DES results - AMR grid & 1.19 \\
\hline
\end{tabular}

Table 1: Comparison of experimental and computational substructure helix traces (from Ref. [31]).
Fig. 6 depicts the power spectral density analysis of the baseline grid solution and the AMR grid solution generated by MATLAB's "psd" function. As is evident in Fig. 6, there is a sensitivity of the resultant frequencies and power to the grid. There is a very dominant frequency occurring at a Strouhal number of approximately 8 , when nondimensionalized by the root chord and the freestream velocity. Both the baseline grid and the AMR grid solutions show a dramatic peak at this Strouhal number with only a small change in the peak with grid density. This frequency has been attributed to the trailing edge vortices $^{2}$. It is also important to notice the range of frequencies around this strong peak in the Strouhal range (from 4 to 30) has an increased power for the AMR grid, indicating additional scales are being captured rather than modeled. It is exactly this behavior that the DES method was designed to capture. As grid density is improved in the focus region, additional scales of eddies are captured resulting in a more detailed simulation. It is important to note that if the purpose of the simulation were to capture phenomenon with a Strouhal number of 6 (as an example) for some multi-disciplinary application such as aeroelasticity, the AMR grid would be more likely to capture the phenomenon accurately.

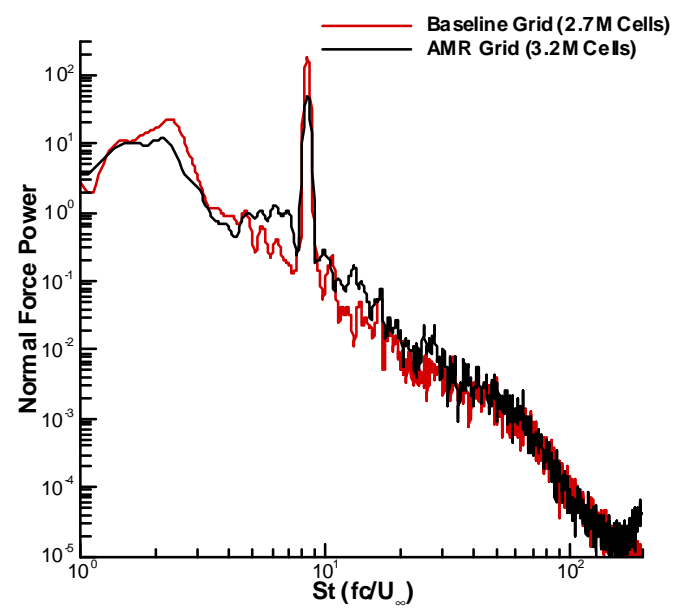

Figure 6: MATLAB power spectral density analysis of the unsteady normal force for the baseline and AMR grids.

\section{F-18C}

All F-18C cases were run at $30^{\circ}$ angle-of-attack, a Mach number of 0.2755 , and a standard day at 20,000 feet. The resulting Reynold's number was 13.9 million based on the mean aerodynamic chord. The baseline grid of 5.9 million cells was generated with VGRIDns after starting from a geometry file provided by Cobalt Solutions LLC from their F-18C challenge work. Steady SA-RANS, unsteady SA-RANS, and unsteady SADES turbulence model simulations were performed on the baseline grid. A timeaveraged SADES solution was used to produce an AMR grid with 6.2 million cells by following the approach outlined for the delta wing above. All time-accurate simulations were run for over 10,000 iterations with second-order temporal and spatial accuracy, three Newton sub-iterations, and a time step of 0.0005 seconds. The 
steady SA-RANS simulation was run for 3000 iterations with first-order temporal and second-order spatial accuracy, one Newton sub-iteration, and a CFL number of 1 milion.

Fig. 7 depicts a top view of the surface mesh and Fig. 8 depicts a cross-plane at a station 410 inches aft of the origin for both the baseline grid and the AMR grid. It is obvious from Fig. 8 that the AMR grid has enhanced resolution in the core of the LEX vortex, the separated region over the wing, at the wingtip pylons, and the under wing pylons. These enhanced grid regions are due to the AMR based on a vorticity iso-surface corresponding to separation regions at these locations and due to the vorticity in the LEX vortex core.

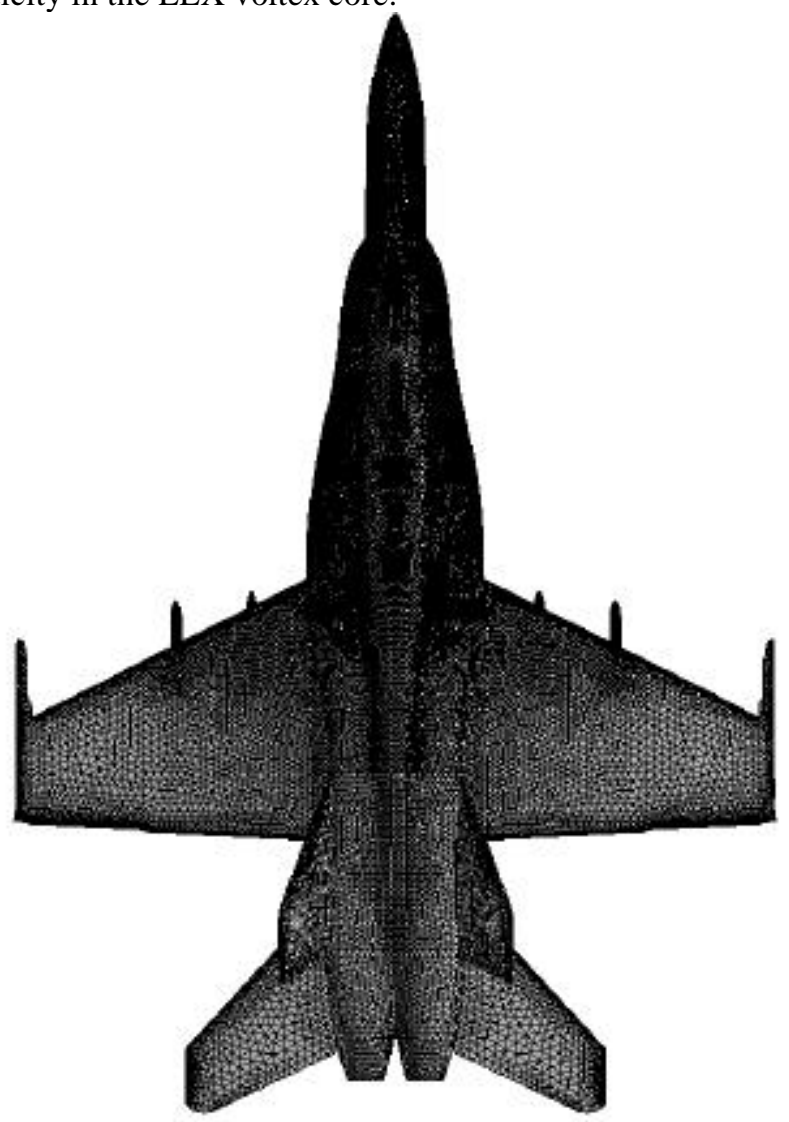

Figure 7: Top view of the baseline grid (5.9 million cells).

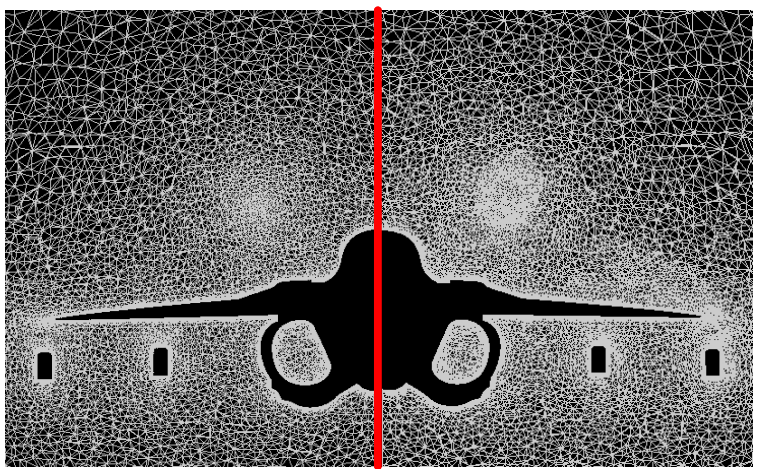

Figure 8: Baseline grid of 5.9 million cells (left) and AMR grid of 6.5 million cells (right) at a station 410 inches aft of the origin.
In Fig. 9, cross-planes of streamwise vorticity at two streamwise locations are shown for the baseline grid solution (left) and the AMR grid solution (right) for a particular instant in time. For ease of comparison, the sense of the vortex is redefined to match the color scheme on the left wing with the right wing even though these vortices rotate in opposite directions. The pre-breakdown cross-plane at 360 inches aft of the origin shows that the AMR grid solution provides better definition of the complex vortical flowfields encountered at this angle of attack. A primary LEX vortex core is observed (red) over the top of a secondary vortex (blue), as well as a wing vortex (blue) is observed just outboard of the LEX secondary vortex. Neither this wing vortex nor the LEX vortex are as well defined on the baseline grid as the AMR grid at this pre-breakdown location. Since the flowfield aft of breakdown varies tremendously with time and the crossplanes at station $410 \mathrm{in}$. are instantaneous, no conclusions can be made for the accuracy of the AMR grid solution versus the baseline grid solution.
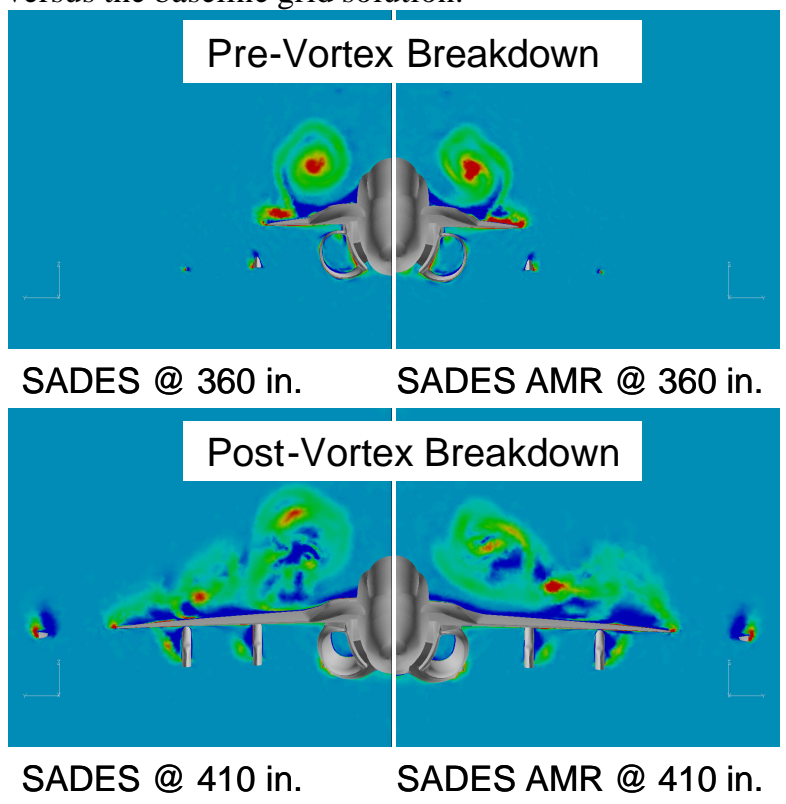

Figure 9: Cross-planes of vorticity at two stations(preand post-breakdown) on the F-18C for the baseline grid (left) and the AMR grid (right).

To determine the location of vortex breakdown for the F$18 \mathrm{C}$ at $30^{\circ}$ angle-of-attack, the streamwise velocity component along the core of the vortex is plotted versus the streamwise location (Fig. 10). A common definition of vortex breakdown is the location where the streamwise velocity component is zero in the core. It should first be noted that both the steady and unsteady SA-RANS simulations produced no vortex breakdown. This inability of commonly used turbulence models to compute a solution with breakdown is well documented in the literature and is due to the large amount of eddy-viscosity these models put into the core of vortices ${ }^{22}$. Several researchers have proposed fixes to these turbulence models by incorporating some form of a rotation correction. The disadvantage of this approach is the fact the simulation 
will still be operating in a RANS mode and compute solutions that are relatively steady post-breakdown as opposed to an LES approach that resolves the eddies that produce the unsteadiness. It is clear in Fig. 10 that the DES method does not suffer from the same problem as the RANS method due to the fact that eddy viscosity is computed based on sub-grid scale turbulence, automatically minimizing the amount of spurious eddyviscosity that is placed in the core of vortices. The baseline grid solution shows vortex breakdown occurring at 430 inches aft of the origin and the AMR grid shows vortex breakdown occurring at 475 inches aft of the origin. It should be noted that these are instantaneous solutions and the vortex breakdown position can vary up to $10 \%$.

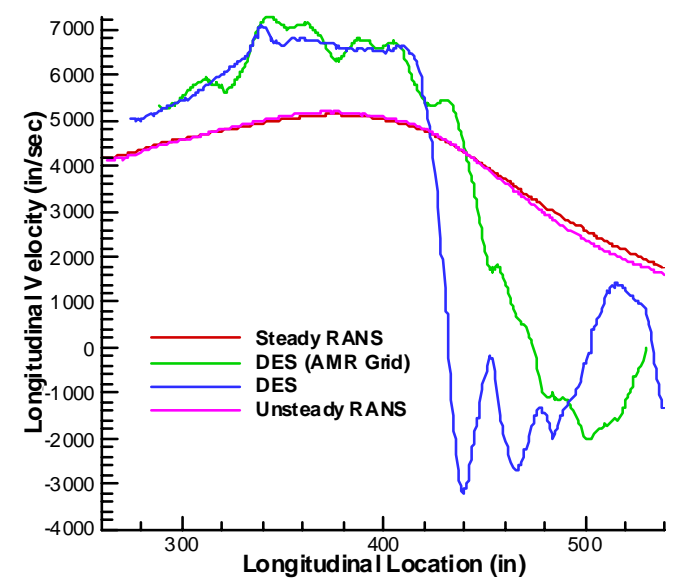

Figure 10: Streamwise velocity component along the LEX vortex core for the SA-RANS and SADES solutions on the baseline grid as well as the SADES solution on the AMR grid.

Fig. 11 depicts the power spectral density (PSD) plot of an outboard tail pressure port. Fig. 11 again shows the inability of standard RANS methods to compute the unsteady flowfield necessary to provide realistic loads data to be used in an aeroelastic analysis. A five order of magnitude increase in power is observed for he DES solutions as compared to the RANS solution. Although the difference is not as dramatic between the baseline grid SADES solution and the AMR grid SADES solution, there is still a respectable improvement in power for the frequency range 0.8 to 8 and the modest increase in cells $(\sim 5 \%)$ of the AMR grid.

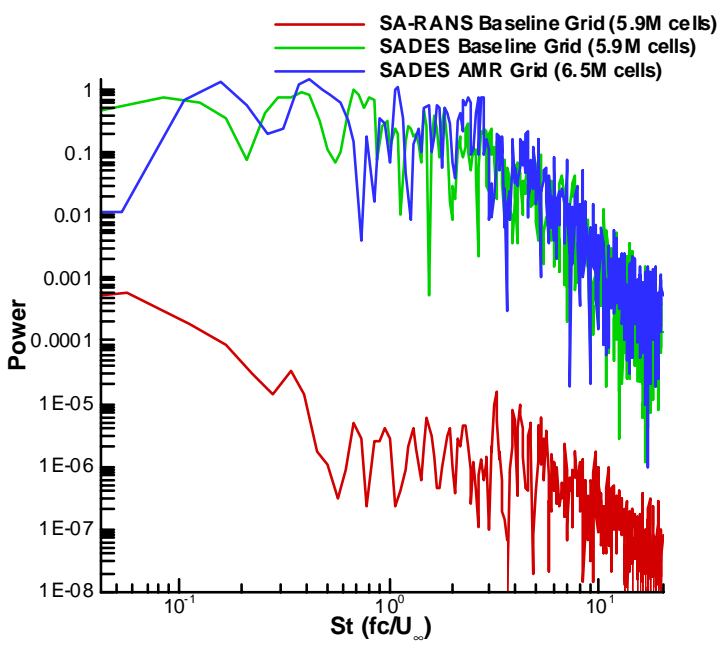

Figure 11: MATLAB power spectral density analysis of the outboard tail pressure port at the 10\% chordwise and $50 \%$ spanwise position for the SA-RANS and SADES baseline grid solution and the SADES AMR grid solution.

Fig. 12 shows an isometric view of the F-18C with an isosurface of vorticity equal to $750 \mathrm{~s}^{-1}$ colored by pressure for the unsteady SA-RANS, SADES baseline grid, and SADES AMR grid solutions. Both the instantaneous solutions and the time-averaged solutions after 10,000 iterations are provided. In Fig. 12 a) and b) it is apparent that the SA-RANS solution does not physically represent the vortex observed in flight and depicted in Fig. 1 for the F-18 HARV. It is also interesting to note the instantaneous and time averaged solutions are essentially equivalent for the SA-RANS solution. On the other hand, both SADES solutions are in excellent qualitative agreement with the vortex breakdown observed in Fig. 1. The SADES solutions also capture the separation over the wing and horizontal tail. Careful examination of Figs. 12 e) and f) show that the AMR grid solution captures small scale structures surrounding the LEX vortex better, consistent with the earlier delta wing case. Figures 12 d) and f) display the time average of the SADES solutions. Both figures show evidence of the vortical substructures in the time average solution. Also, the time average of the AMR grid shows an iso-surface that extends further aft. By compiling a series of these snapshots, a movie can be created that shows the unsteady behaviour of the postbreakdown windings, the pre-breakdown substructures, and the separated flow regions. It is clear from the simulation that the tails are in a very unsteady environment contributing to the fatigue issues well documented for the F-18 without the LEX fence. It is also clear that the industry standard RANS methods for these high Reynolds number flows are completely inadequate for obtaining unsteady loads on tails due to vortex breakdown. 

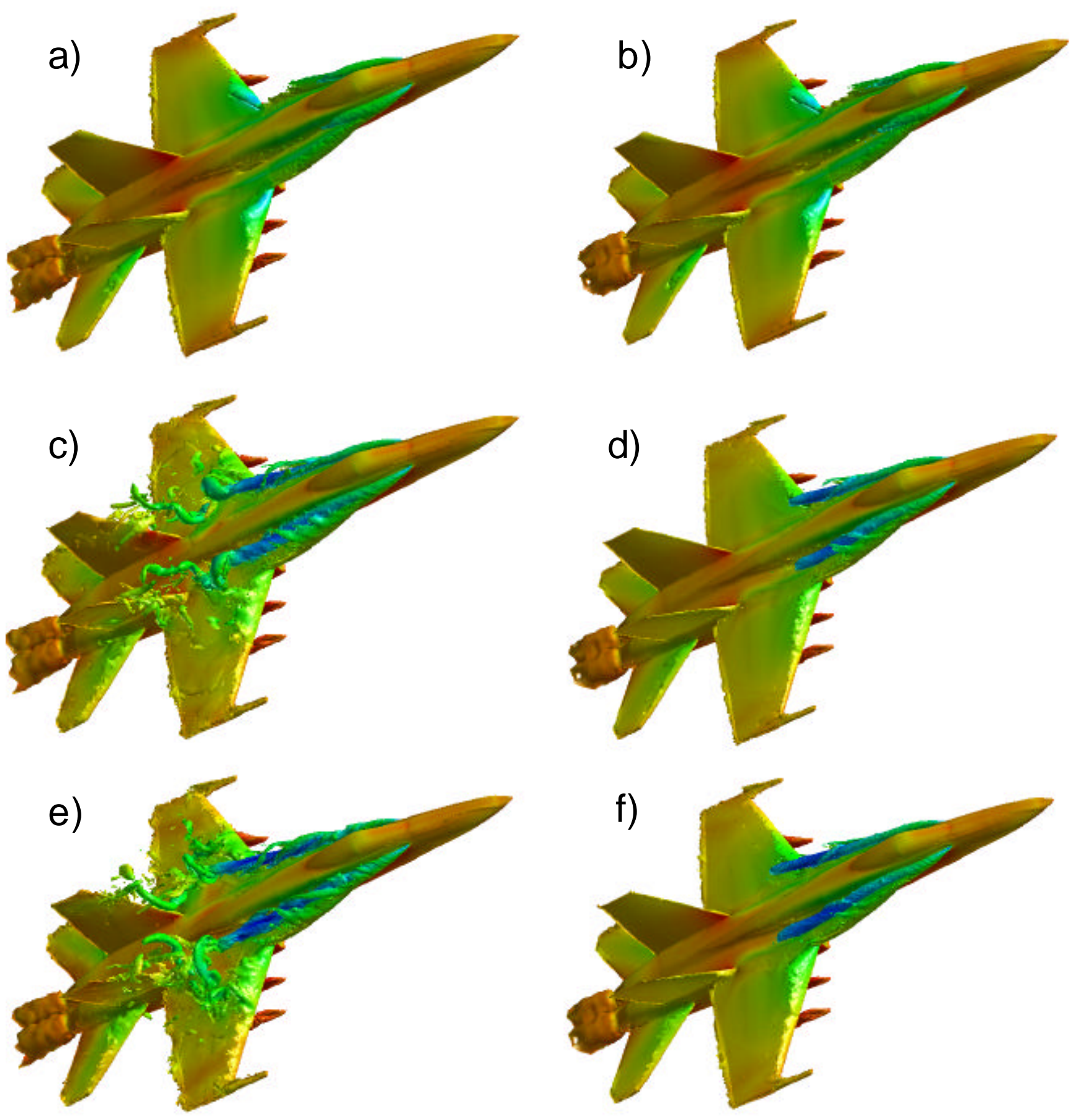

\section{Instantaneous}

Figure 12: Isometric views of the F-18C at $\alpha=30^{\circ}, \operatorname{Re}_{c}=$ $13.9 \times 10^{6}$, leading and trailing edge flaps set to $0^{\circ}$ and the diverter slot present. a), c), and e) depict instantaneous views of the SA-RANS, SADES baseline grid, and SADES $A M R$ grid solution, respectively. $b$ ), $d$ ), and f) depict solutions time averaged after 10,000 time steps for the the SA-RANS, SADES baseline grid, and SADES AMR grid solution, respectively. 
In order to lend credibility to the F-18C SADES solutions with and without AMR of the current study, comparison is made with the F-18 HARV flight test data ${ }^{3,4,42}$. It is important to note the differences in the $\mathrm{F}-18 \mathrm{C}$ grid and the actual F-18 HARV. The F-18C of the current study has leading edge flaps set to 0 degrees deflection whereas the F-18 HARV leading edge flaps were deflected down 33 degrees. The trailing edge flaps were set to 0 degrees deflection for both the F-18C and the F-18 HARV. The F$18 \mathrm{C}$ has a diverter slot that goes through the upper surface of the LEX creating a jet-like flowfield above the LEX but was sealed over for the F-18 HARV. Also, the under-wing pylons are on the $\mathrm{F} 18 \mathrm{C}$ but were taken off of the F18 HARV. Finally, the F-18C has rigid tails in the simulations but they are fairly flexible in the F18 HARV with tip deflections on the order of a few percent of the tail root chord. Although these differences in configuration are not trivial, comparison can still be made to determine the qualitative agreement with the flight test.

Fig. 13 displays the lift force in pounds of the simulation as a function of time. The steady and unsteady RANS simulations converge quickly to the same lift force of $37,175 \mathrm{lbs}$ with a variation of plus or minus $20 \mathrm{lbs}$ and both SADES solutions have a mean of $35,400 \mathrm{lbs}$ plus or minus 1,800 lbs. Therefore, the SA-RANS simulations are $5 \%$ higher than the SADES solutions and relatively steady. If the $\mathrm{F}-18 \mathrm{C}$ grid was modified to include the $-33^{\circ}$ leadingedge flap extension, both the SA-RANS and SADES simulations would have a higher lift, due to the increased camber of the wing, possibly moving the SADES solutions closer to the flight test. It is interesting to note that the F18 HARV was fairly trimmed with a weight of $37,193 \mathrm{lbs}$, indicating that the simulations are qualitatively correct.

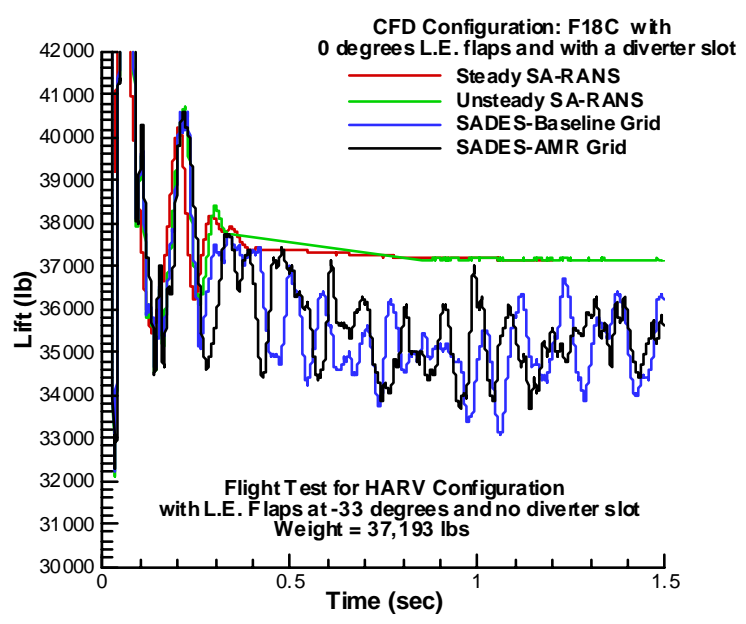

Figure 13: Lift as a function of time for the F-18C at $\alpha=$ $30^{\circ}, R_{c}=13.9 \times 10^{6}$, leading and trailing edge flaps set to $O^{\circ}$ and the diverter slot present. Steady SA-RANS, unsteady SA-RANS, and unsteady SADES solutions are provided for the baseline grid and an SADES solution is provided for the AMR grid.
Fig. 14 shows the PSD of the F-18 HARV outboard vertical tail pressure port in the same location as the simulation pressure port of Fig. 11. Although the power is not of the same magnitude, the frequency roll off is very similar to the SADES grid solutions presented in Fig. 11. The Strouhal frequency at the peak of both the SADES and flight test is approximately equal to 1 .

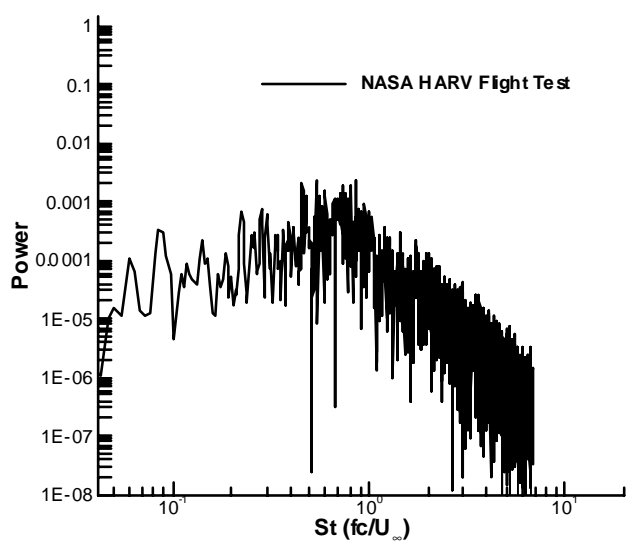

Figure 14: MATLAB power spectral density analysis of the outboard tail pressure port at the 10\% chordwise and 50\% spanwise position for flight 198 of the NASA HARV F-18.

Finally, Fig. 15 is a well known plot in the literature of the streamwise location of the LEX vortex breakdown as a function of angle-of-attack ${ }^{4}$. For the $30^{\circ}$ angle-of-attack of interest in this study, vortex breakdown occurs between $40 \%$ and $50 \%$. The location of breakdown observed in Fig. 10 for the simulations is at $60 \%$. This discrepancy is not surprising when considering the fact that the diverter slot is covered up on the F-18 HARV. Mitchell et. al. ${ }^{17}$ demonstrated that along the core blowing from the surface can move the breakdown position aft. The jet-like behavior of the diverter slot could be acting like a vortex breakdown flow control device, explaining the aft position of vortex breakdown for the F-18C with a diverter slot.

\section{CONCLUSIONS \& RECOMMENDATIONS}

Detached eddy simulations have been provided for a $70^{\circ}$ delta wing using a baseline grid and an adaptive mesh refinement grid. The adaptive mesh refinement grid solution was shown to capture the smaller scale features present in the experiment but not captured by the baseline grid. In addition, the AMR grid solution showed an increase in the related power over the baseline grid for a range of frequencies giving evidence that an increase in the number of small scale features were being resolved over the baseline grid. The AMR grid solutions also compared very well to the helix measurement of the vortical substructures and the vortex breakdown position determined by the Onera experiments.

The combined SADES and AMR grid approach demonstrated on the delta wing was then applied to an $\mathrm{F}$ $18 \mathrm{C}$ to determine if the unsteady tail loads could be 
simulated. As in the delta wing case, the AMR F-18C grid SADES solution showed an improvement in capturing small scale features of the LEX vortex as compared to the baseline grid SADES solution. Also, an improvement in the power associated with a range of frequencies was demonstrated for the SADES AMR grid solution over the baseline grid solution, consistent with the delta wing analysis. In all cases the SA-RANS solutions proved completely inadequate for computing vortex breakdown for a flight vehicle at high Reynolds number.

Flight test data from the NASA HARV program was used to show the solutions were reasonable even though the aircraft configurations were different. Qualitative agreement between the SADES solutions and the HARV data was obtained for the lift and the vortex breakdown position. A recommendation for future research is to create an F-18C grid that is a closer match to the HARV by moving the leading-edge flap to a $-33^{\circ}$ position, close off the diverter slot, and eliminate the under-wing pylons.

\section{ACKNOWLEDGEMENTS}

The authors would like to say thanks to Dr Shahyar Pirzadeh for his help with the AMR software, Dave Fisher and Al Bowers of the NASA Dryden Flight Research Center for their invaluable assistance with the F-18 HARV flight test data, and Maj Tony Mitchell of the USAF Academy for his help with the Onera experiments of the $70^{\circ}$ delta wing. This work could not have been accomplished without the support of the ASC Major Shared Resource Center of HPCMO and the USAFA Modeling \& Simulation Resource Center. Finally, the authors would like to express their gratitude for the support of this project by Dr John Schmisseur of AFOSR.

\begin{tabular}{|c|c|c|c|c|}
\hline & $\mathrm{R}_{\mathbf{c} \overline{\mathbf{c}}}$ & $\begin{array}{c}\text { Model scale, } \\
\text { percent }\end{array}$ & Fluid medium & Rot. \\
\hline - Flight & $81013 \times 10^{6}$ & & Air & 18 \\
\hline ] отRс & $1.75 \times 10^{6}$ & 6 & Air & 4,18 \\
\hline 0 BART & $1.60 \times 106$ & 3 & Air & 18 \\
\hline A LSWT & $3.60 \times 10^{5}$ & 12 & Air & 18 \\
\hline$\nabla$ FVF & $1.26 \times 10^{4}$ & 3 & Water & 18 \\
\hline
\end{tabular}

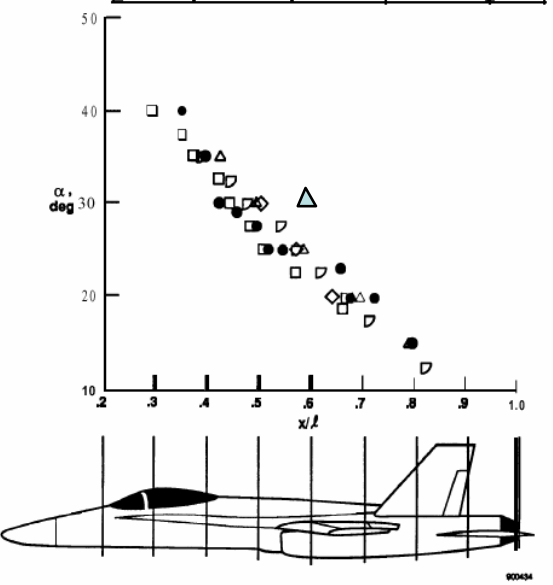

Figure 15: Streamwise LEX vortex breakdown position as a function of angle of attack extracted from Ref.[4]. SADES AMR grid solution vortex breakdown location plotted with the flight test and experimental data for comparison.
${ }^{1}$ Lovell, D A, “Military Vortices”, Research \& Technology Organization Applied Vehicle Technology Panel Meeting, Norway, 7-11 May 2001.

${ }^{2}$ Morton, S.A., Forsythe, J.R., Mitchell, A.M., and Hajek, D., "DES and RANS Simulations of Delta Wing Vortical Flows," AIAA 2002-0587.

${ }^{3}$ Lee, B.H.K., "Vertical Tail Buffeting of Fighter Aircraft," Progress in Aerospace Sciences 36 (2000) 193-279.

${ }^{4}$ Fisher, D., Del Frate, J.H., and Zuniga, F.A., "Summary of In-Flight Flow Visualization Obtained From the NASA High Alpha Research Vehicle,” NASA TM-101734, January 1991.

${ }^{5}$ Murman, S.M., Rizk, Y.M, Cummings, R.M., and Schiff, L.B., "Computational Investigation of Slot Blowing for Fuselage Forebody Flow Control," Aircraft Design, Vol. 2, 1999, pp. 4563.

${ }^{6}$ Werlé, H., "Quelques résultats expérimentaux sur les ailes en flèche, aux faibles vitesses, obtenus en tunnel hydrodynamique" La Recherche Aéronautique, No. 41, Sep.-Oct. 1954, pp. 15-21.

${ }^{7}$ Peckham, D.H., Atkinson, S.A., "Preliminary Results of Low Speed Wind Tunnel Tests on a Gothic Wing of Aspect Ration 1.0", Aeronautical Research Council Technical Report, C.P. No 508, T.N. Aero 2504, Apr. 1957.

${ }^{8}$ Elle, B.J., "An Investigation at Low Speed of the Flow near the Apex of Thin Delta Wings with Sharp Leading Edges", Aeronautical Research Council R\&M, No. 3176, 1961.

${ }^{9}$ Lambourne, N.C., Bryer, D.W., "The Bursting of Leading-Edge Vortices-Some Observations and Discussion of the Phenomenon", Aeronautical Research Council R\&M No. 3282, 1962.

${ }^{10}$ Hall, M.G., "Vortex Breakdown", Annual Review of Fluid Mechanics, Vol. 4, 1972, pp. 195-218.

${ }^{11}$ Leibovich, S., "The Structure of Vortex Breakdown," Annual Review of Fluid Mechanics, Vol. 10, 1978, pp. 221-46.

${ }^{12}$ Sarpkaya, T. "On Stationary and Traveling Vortex Breakdowns," Journal of Fluid Mechanics, Vol. 45, Part 3, 1971, pp. 545-559.

${ }^{13}$ Nelson, R.C., "Unsteady Aerodynamics of Slender Wings," Aircraft Dynamics at High Angles of Attack: Experiments and Modeling, AGARD-R-776, 1991, pp.1-1 to 1-26.

${ }^{14}$ Délery, J., "Aspects of Vortex Breakdown," Progress in Aerospace Sciences, Vol. 30, 1994, pp. 1-59.

15 Faller, J.H., Leibovich, S., "Disrupted states of vortex flow and vortex breakdown", Physics of Fluids, Vol. 20, No. 9, 1977, pp.1385-1400.

${ }^{16}$ Menke, M., Yang, H., Gursul, I., "Further Experiments on Fluctuations of Vortex Breakdown Location," AIAA-96-0205, $34^{\text {th }}$ AIAA Aerospace Sciences Meeting and Exhibit, Jan 1996, Reno, NV.

${ }^{17}$ Mitchell, A.M., Molton, P., Barberis, D., Délery, J., "Oscillation of Vortex Breakdown Location and Control of the Time-Averaged Location by Blowing," AIAA Journal, Vl. 38, No. 5, May 2000, pp.793-803. 
${ }^{18}$ Spalart, P. R., and Allmaras, S.R., "A One Equation Turbulence Model for Aerodynamic Flows," La Recherche Aerospatiale, 1994, 1, p.5.

${ }^{19}$ Spalart, P., "Young-Person's Guide to Detached-Eddy Simulation Grids," NASA CR 2001-211032.

${ }^{20}$ Strang, W.Z., Tomaro, R.F., Grismer, M.J., "The Defining Methods of Cobalt: A Parallel, Implicit, Unstructured Euler/Navier-Stokes Flow Solver," AIAA 99-0786, January 1999.

${ }^{21}$ Forsythe, J.R., Hoffmann, K.A., Cummings, R.M., Squires, K.D.., "Detached-Eddy Simulation with Compressibility Corrections Applied to a Supersonic Axisymmetric Base," Journal of Fluids Engineering, Vol. 124, No. 4, 2002, pp. 911-923.

${ }^{22}$ Morton, S.A., Forsythe, J.R., Mitchell, A.M., and Hajek, D., "Detached-Eddy Simulations and Reynolds-Averaged NavierStokes Simulations of Delta Wing Vortical Flowfields," Journal of Fluids Engineering, Vol. 124, No. 4, 2002, pp. 924-932.

${ }^{23}$ Squires, K.D., Forsythe, J.R., and Spalart, P.R., "Detached-Eddy Simulation of the Separated Flow Around a Forebody CrossSection," Direct and Large Eddy Simulation IV, ERCOFTAC Series - Volume 8, B.J. Geurts, R. Friedrich and O. Metais, editors, Kluwar Academic Press, pp. 481-500, 2001.

${ }^{24}$ Forsythe, J.R., Squires, K.D., Wurtzler, K.E. and Spalart, P.R., "Detached-Eddy Simulation of Fighter Aircraft at High Alpha", AIAA 2002-0591, January 2002.

${ }^{25}$ Forsythe, J.R., Woodson, S.H., "Unsteady CFD Calculations of Abrupt Wing Stall Using Detached-Eddy Simulation", AIAA 2003-0594, Jan 2003.

${ }^{26}$ Samareh, J., "Gridtool: A Surface Modeling and Grid Generation Tool," Proceedings of the Workshop on Surface Modeling, Grid Generation, and Related Issues in CFD Solution, NASA CP3291, May 9-11, 1995.

${ }^{27}$ Pirzadeh, S., "Progress Toward A User-Oriented Unstructured Viscous Grid Generator," AIAA Paper 96-0031, January 1996.

${ }^{28}$ Spalart, P. R., Jou W-H. , Strelets M. , and Allmaras, S. R., "Comments on the Feasibility of LES for Wings, and on a Hybrid RANS/LES Approach," Advances in DNS/LES, 1st AFOSR Int. Conf. on DNS/LES, Aug 4-8, 1997, Greyden Press, Columbus Oh.

${ }^{29}$ Forsythe, J.R., Hoffmann, K.A., Dieteker, F.F., "Detached-Eddy Simulation of a Supersonic Axisymmetric Base Flow with an Unstructured Flow Solver," AIAA 00-2410, June 2000.

${ }^{30}$ Morton, S.A., Forsythe, J.R., Squires, K.D., and Wurtzler, K.E., "Assessment of Unstructured Grids for Detached-Eddy Simulation of High Reynolds Number Separated Flows," $8^{\text {th }}$ ISGG Conference, Honolulu, June 2002.

${ }^{31}$ Mitchell, A., Morton, S., and Forsythe, J., "Analysis of Delta Wing Vortical Substructures Using Detached-Eddy Simulation," AIAA-2002-2968, June 2002.

${ }^{32}$ Pirzadeh, S., "'Vortical Flow Prediction Using an Adaptive Unstructured Grid Method", Research \& Technology Organization Applied Vehicle Technology Panel Meeting, Norway, 7-11 May 2001.
${ }^{33}$ Squire, H.B., Jones, J.G., and Stanbrook, A., “An Experimental Investigation of the Characteristics of Some Plane and Cambered 65 Deg Delta Wings at Mach Numbers from 0.7 to 2.0," ARC, R\&M No. 3305, 1961.

${ }^{34}$ Payne, F.M., Ng, T.T., Nelson, R.C. and Schiff, L.B., "Visualization and Wake Surveys of Vortical Flow over a Delta Wing," AIAA Journal, Vol. 26, No. 2, February 1988, pp 137143.

${ }^{35}$ Lowson, M.V., "Visualization Measurements of Vortex Flows," AIAA Paper 89-0191, Jan. 1989.

${ }^{36}$ Veerhagen, N.G., Meeder, J.P., and Verhelst, J.M., "Boundary Layer Effects of the Flow of a Leading-Edge Vortex," AIAA Paper 93-3463, August 1993.

${ }^{37}$ Washburn, A.E. and Visser, K.D., "Evolution of Vortical Structures in the Shear Layer of Delta Wings," AIAA Paper 94 2317, June 1994.

${ }^{38}$ Lowson, M.V., Riley, A.J., and Swales, C., "Flow Structure Over Delta Wings," AIAA Paper 95-0586, January 1989.

${ }^{39}$ Honkan, A. and Andreopoulos, J., "Instantaneous ThreeDimensional Vorticity Measurements in Vortical Flow Over a Delta Wing," AIAA Journal, Vol. 35, No. 10, Oct. 1997, pp. 1612-1620.

${ }^{40} \mathrm{Ng}$, T.T. and Oliver, D.R., "Leading-Edge Vortex and Shear Layer Instabilities,” AIAA Paper 98-0313, January 1998.

${ }^{41}$ Mitchell, A.M. and Molton, P., "Vortical Substructures in the Shear Layers Forming Leading-Edge Vortices," AIAA Paper 2001-2424, June 2001.

${ }^{42}$ Ames Research Centert Dryden Flight Research Facility, "High Alpha Research Vehicle Phase II Flight Report, Flight 197199," HA94-70-660, February 1994. 